\title{
Diagnosis of Bearing Defects Using a Heterodyning Ultrasound Detector
}

\author{
Stuart Taylor $\cdot$ Kirk Wiggins
}

Submitted: 14 May 2015 / Published online: 3 July 2015

(C) ASM International 2015

\begin{abstract}
A single row ball bearing was presented to the Failure Analysis Lab because of noise and perceived roughness during low speed operation. The bearing was analyzed using a heterodyning ultrasound detector. Discrete sources of intermittent noise were detected. A quasi-static determination of the friction coefficient of the bearing was made. Contamination by hard foreign objects was suspected. The bearings were solvent washed, and particles of aluminum were recovered. The size of these particles was related to the static friction coefficient of the bearing. The cleaned bearings were retested and found to have residual friction related to microscopic defects caused by inclusions and voids in the races and balls. A generalized theory was formulated to account for the relation between noise and static friction.
\end{abstract}

Keywords Rolling element bearing .

Heterodyning ultrasound B Bearing roughness .

Bearing inclusions $\cdot$ Bearing steel metallurgy

\section{Background}

The sound produced by a ball bearing can be traced to defects and/or solid foreign objects that interfere with the rolling of a ball over a race surface [1-4]. The human ear is not capable of hearing the high frequency sounds generated by impacts between ball and race as the rolling contact between them is periodically impeded. However, the human fingertip is remarkably adept at detecting small defects as the bearings

S. Taylor $\cdot$ K. Wiggins $(\bowtie)$

Failure Analysis Laboratory, Raytheon Systems Company,

McKinney, TX, USA

e-mail: kwiggins@raytheon.com are rotated slowly by hand. Thus, the failure analysis lab receives bearings for failure analysis because of a qualitative, perceived roughness or grittiness. In applications where sensors are supported by bearings, the production of any noise or roughness at low speed rotation is a concern. So, in these applications, having a way of characterizing objectionable sounds or grittiness in low speed operation is desired. Merely amplifying the sound will not clarify the high frequency inaudible sounds made by the bearing. The sounds must be made audible by the heterodyning process which converts inaudible high frequency sounds to audible sounds within the human ear's audible range. A high frequency noise is ideally multiplied (mixed) by a constant frequency reference signal which is close to the signal to be detected. According to the trigonometric identity

$$
\begin{aligned}
\sin \left(\omega_{\mathrm{n}} t\right) \sin \left(\omega_{\mathrm{r}} t\right)= & 1 / 2 \cos \left[\omega_{\mathrm{n}} t-\omega_{\mathrm{r}} t\right]-1 / 2 \cos \left[\omega_{\mathrm{n}} t\right. \\
& \left.+\omega_{\mathrm{r}} t\right]
\end{aligned}
$$

where $\omega_{\mathrm{n}} t$ is the noise frequency and $\omega_{\mathrm{r}} t$ is the reference frequency [5].

If the noise and reference signal are close to the same, the difference of the two frequencies in the first term of the above equation results in a low frequency audible signal. This process is shown graphically below.

The heterodyned signal is the low frequency component of the sum of the unknown signal and a constant reference signal of similar frequency. It has a lower frequency than either and can be heard by the human ear. This process is a great advantage in routine evaluation of rotating machinery and perceived rough bearings.

Using this process in the laboratory, the investigator can vary the reference frequency by trial and error while listening to the subject bearing operating at low speed. As the 
reference frequency gets close to the unknown signal frequency, the operator hears a series of amplitude spikes or "pops" as the bearing surfaces collide with a defect.

\section{Instantaneous Torque}

In this application, the ball bearing is operated at low speed and low load. It is used to provide low-friction rotation for a tracking system. Any irregularity in motion is a serious matter in the tracking ability of the system supported by this bearing (Fig. 1).

During operation, the rolling elements encounter defects which result in a momentary increase in the coefficient of rolling friction. These defects are inherent in the bearing material or imposed in the form of contaminating particles from the environment. These defects reveal themselves as momentary peaks in the ultrasound signature of the bearing during low speed operation $[2,3]$. In either case, the frictional effect of the irregularity may be calculated as follows (Figs. 2, 3, 4).

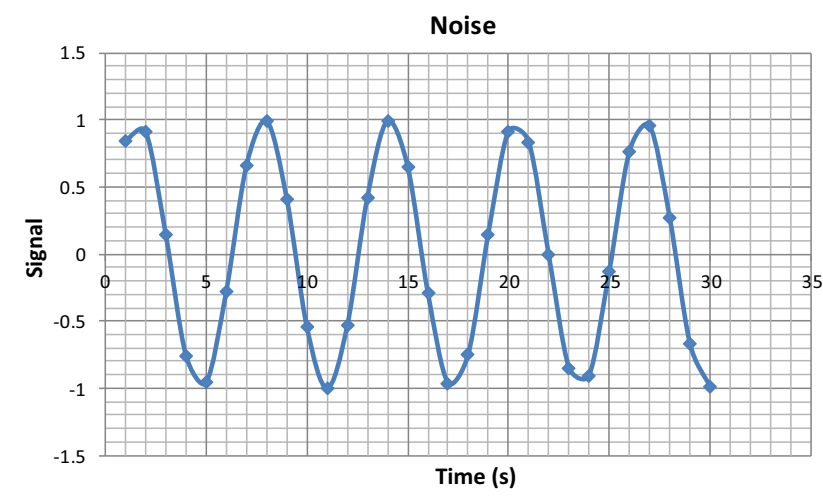

Fig. 1 Hypothetical high frequency noise signal for the heterodyning process

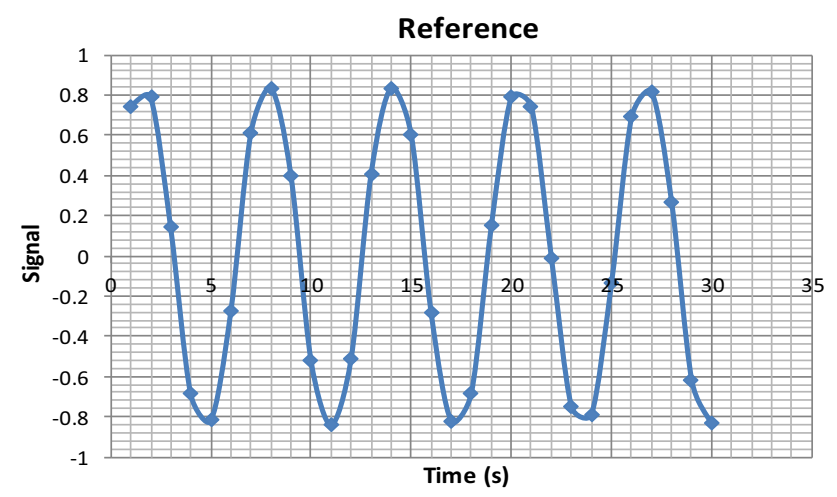

Fig. 2 Hypothetical reference signal for the heterodyning process
Consider a bearing ball rolling over an obstruction as the inner race has a moment $M$ exerted. Since this rotation is low speed, assume a quasi-equilibrium condition and write the static moment balance about the point of contact between the ball and the obstruction of height $h$ (Fig. 5).

The static equilibrium about the contact point is defined as $L *\left(2 h r-h^{2}\right)^{\frac{1}{2}}-F_{\mathrm{t}} *(2 r-h)=0$

The coefficient of friction is $\mu=\frac{M}{L R}$ where $R \gg r$. Since the moment is $M=F_{\mathrm{t}} R$ and $d=2 r$, the coefficient of friction is defined simply $\mu=(h / d)^{1 / 2}$ (where $\left.h \ll d\right)$.

Note that no sliding or irreversible processes such as plastic yielding of viscous flow are considered in this analysis. Resisting moments in the coefficient of friction calculation are a result of potential energy change only.

\section{Case History}

The primary problem with the bearings as presented was the presence of aluminum chips from a machining operation. These chips do not correspond to any material found in the bearing so their origin is a mystery. Solvent cleaning removed the chips along with accompanying lubrication and resulted in a reduction of friction to about $60 \%$ of the original. However, some "roughness" or periodic friction moment and noise persisted.

The question remains why is there any friction at all in the clean bearings after the contamination is removed? Microscopic examination reveals voids and metallurgical defects on the surface balls and races measured at about 2.75 micron average size. These are not a result of contamination. They are intrinsic to the bearing raw material. Assuming that the microscopic defects are acting as "bumps" to impede motion, the size of the defects should be a predictor of their effect on instantaneous friction (Fig. 6).

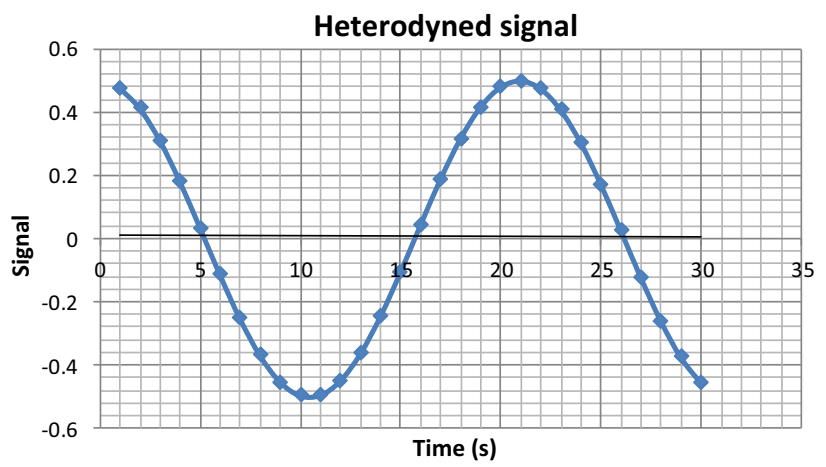

Fig. 3 Heterodyned signal after combining the noise and reference signal 
Fig. 4 Typical bearing noise for low load, low speed operation

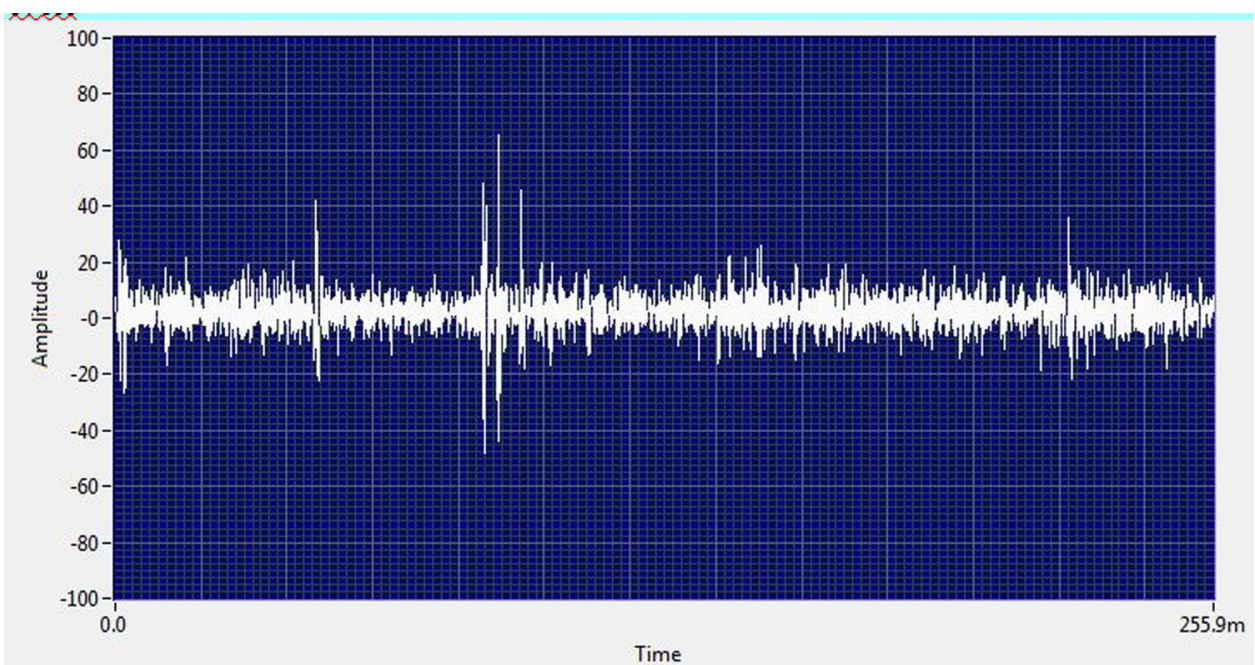

Fig. 5 Low speed ball bearing relation to a defect in the inner race

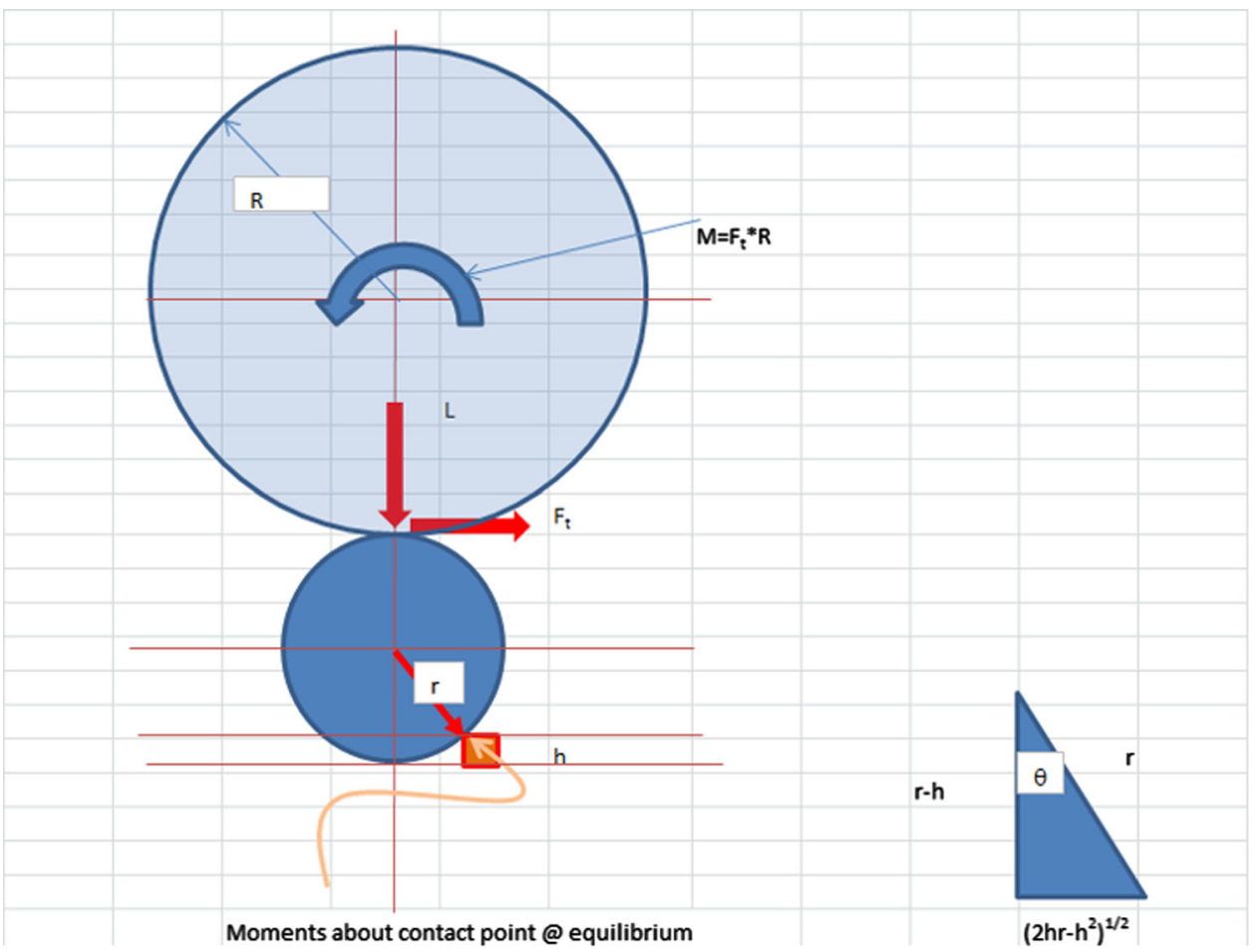

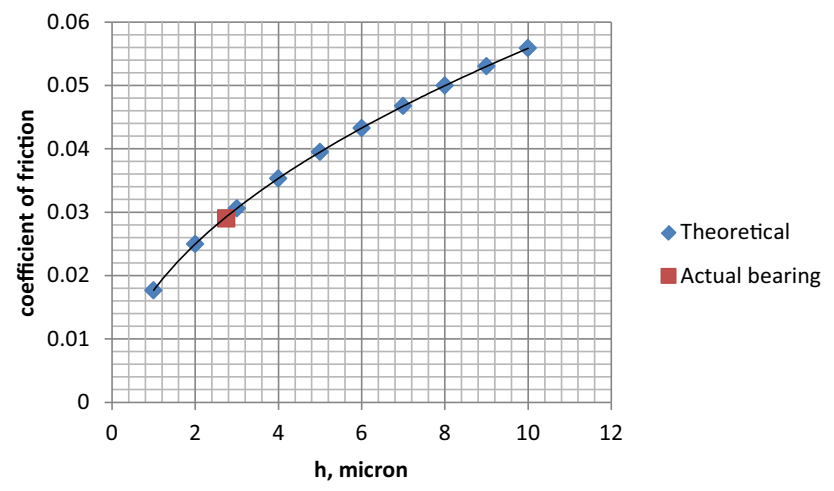

Fig. 6 Theoretical instantaneous friction vs. defect size
An obstruction height of 2.75 microns gives a value for theoretical coefficient of friction $\mathrm{m}$ of $\mathbf{0 . 0 2 9}$, as calculated above. This compares to $\mathbf{0 . 0 2 4}$ average for measured values [6].

Note that this friction is instantaneous rather than average friction. Average friction as measured is typically about $75 \%$ of this value. For this application, the average friction should be improved-published values for average ball bearing friction are typically in the $0.001-0.002$ range. This implies that the raw material as well as the machining processes must be improved to produce a suitable bearing (Fig. 7). 


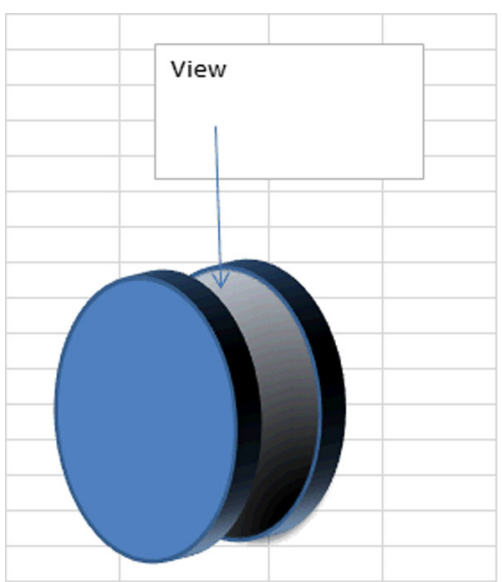

Fig. 7 Normal view of inner race

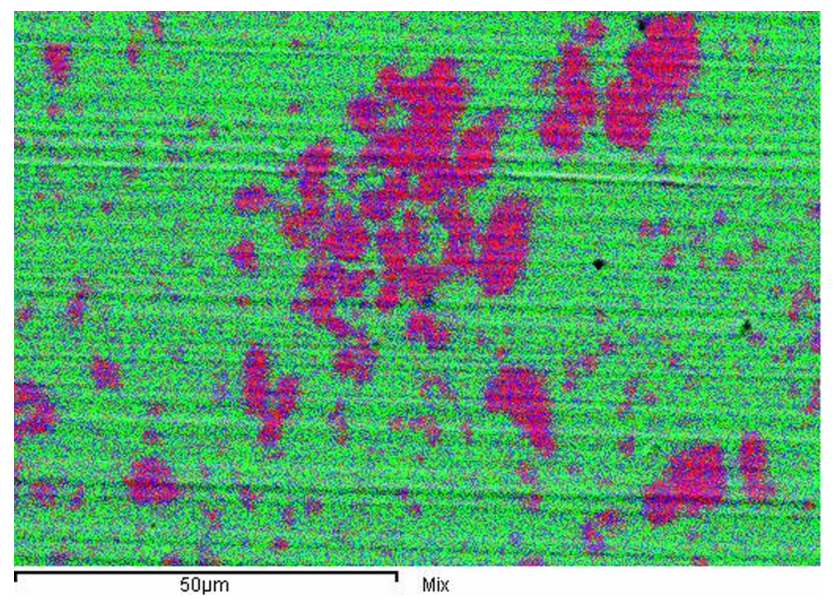

Fig. 8 Surface of the bearing race. The color represents materials: Green $=\mathrm{Fe}$, Blue $=\mathrm{Mn}$, Red $=\mathrm{Cr}$ (Color figure online)

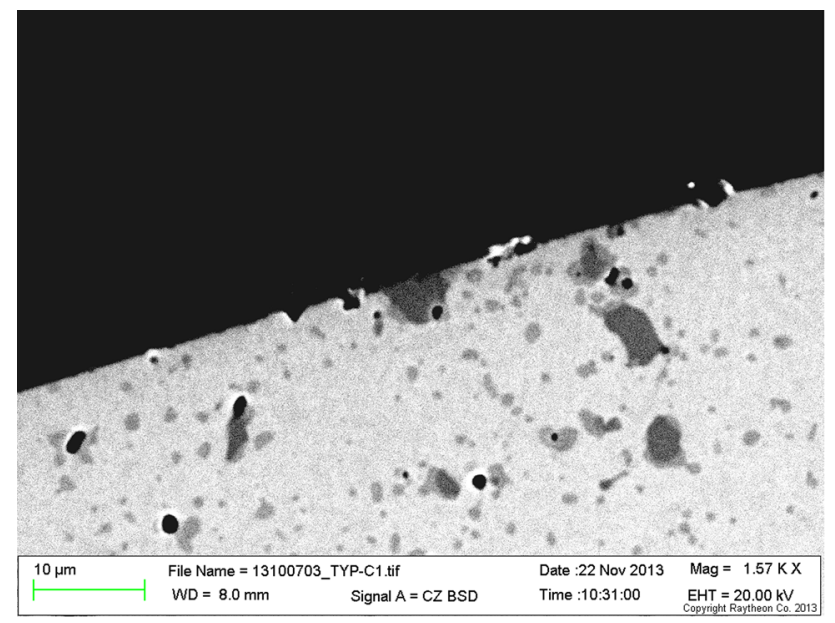

Fig. 9 Typical section through the ball
Recommendations: refer to vendor for cleanliness remediation. Investigate purchase of higher quality bearings to match the application.

\section{Microscopy}

The next phase of this analysis is the correlation of audible defects and coefficient of friction with observable features of the bearing steel (Fig. 8).

Figure 9 is the normal view of the surface of the bearing race. The steel is not homogeneous. $\mathrm{Cr} / \mathrm{Mn}$ phases have been ground through to finish the inner race. These are potential causes of roughness; the properties of the phases are different from the iron matrix [7].

$\mathrm{Fe}-\mathrm{Cr}$ phases are often associated with voids. The size of these voids predicts the instantaneous friction coefficient of the bearing.

\section{Conclusions}

The Ultrasonic heterodyning detector provides a good method for detecting roughness in ball bearings. Friction in ball bearings is associated with metallurgical irregularities and voids.

The value of instantaneous coefficient of friction for lightly loaded bearings depends closely on the defect size discovered by metallographic sectioning. Control of these defects is essential if expected behavior is to be obtained, especially for low speed applications.

\section{References}

1. P.D. McFadden, Vibration monitoring of rolling element bearings by the high-frequency resonance technique-a review. Tribol. Int. 17(1), 3-10 (1984)

2. Y.-H. Kim, et al. Condition Monitoring of low speed bearings: a comparative study of the ultrasound technique versus vibration measurement WCEAM (2006) paper 029

3. A. Morhain, Bearing defect diagnosis and acoustic emission. Proc. Inst. Mech. Eng. Part J 217(4), 257-272 (2003)

4. A. Takeuchi, Diagnostic Technique of Abnormalities in Ball Bearings with an Ultrasonic Method. 12th A-PCNDT 2006Asia-Pacific Conference on NDT, 5th-10th Nov. (Auckland, New Zealand, 2006)

5. C. Cooper, Physics (Fitzroy Dearborn, Chicago, 2001)

6. P. Eschmann, L. Hasbargen, K. Weigand, Ball and Roller Bearings-Theory, Design, and Application. 2nd edn. (Wiley, 1985)

7. H. Bhadeshia, Steels for Bearings. Prog. Mater. Sci. 57, 268-435 (2012). University of Cambridge, Department of Materials Science and Metallurgy 